\title{
Casein kinase $1 \alpha$ inhibits p53 downstream of MDM2-mediated autophagy and apoptosis in acute myeloid leukemia
}

\author{
WANLING XU ${ }^{1 *}$, ZIYANG HUANG $^{1 *}$, YIFENG GAN $^{1}$, RONGRONG CHEN $^{1}$, YISHA HUANG ${ }^{1}$, \\ BIN XUE ${ }^{1}$, SONGFU JIANG ${ }^{1}$, ZHIJIE YU ${ }^{1}, \mathrm{KANG} \mathrm{YU}^{1}$ and SHENGHUI ZHANG ${ }^{1,2}$ \\ ${ }^{1}$ Department of Hematology, Wenzhou Key Laboratory of Hematology, ${ }^{2}$ Division of Clinical Research, \\ The First Affiliated Hospital of Wenzhou Medical University, Wenzhou, Zhejiang 325015, P.R. China
}

Received April 1,2020; Accepted July 8, 2020

DOI: $10.3892 / o r .2020 .7760$

\begin{abstract}
Enhancement of autophagy serves as a promising therapeutic strategy for cancer,including acute myeloid leukemia (AML). Casein kinase $1 \alpha(\mathrm{CK} 1 \alpha)$, encoded by CSNK1A1, regulates $\mathrm{Wnt} / \beta$-catenin, $\mathrm{p} 53$ and other key signaling pathways, and is critically involved in tumor progression. However, the relationship and mechanism of CK1 $\alpha$ with autophagy in AML still remain unclear. In the present study, it was found that AML patients had higher expression of CSNK1A1 mRNA than healthy donors. Furthermore, we analyzed 163 cases of AML patients in the LAML database of TCGA and found that AML patients with high CSNK1Al had shorter overall survival than those with low or medium CSNKIAl expression. Furthermore, we demonstrated that $\mathrm{CK} 1 \alpha$ was a negative regulator of autophagy and apoptosis. Pharmacologic inhibition of CK1 $\alpha$ using D4476 or CK1 $\alpha$ knockdown via lentivirus-mediated shRNA suppressed proliferation and the clone formation by enhancing autophagic flux and apoptosis in AML cell lines as well as in patient blast cells. Intriguingly, D4476-induced cell death was aggravated in combination with an autophagy inhibitor, Spautin-1, suggesting that autophagy may be a pro-survival signaling. CK1 $\alpha$ interacted with murine double minute 2 (MDM2) and p53, and CK1 $\alpha$ inhibitor D4476 significantly upregulated p53 and phosphorylated 5' AMP-activated protein kinase (AMPK), and substantially inhibited the
\end{abstract}

Correspondence to: Professor Kang Yu, Department of Hematology, Wenzhou Key Laboratory of Hematology, The First Affiliated Hospital of Wenzhou Medical University, Shangcai Village, Nanbaixiang Town, Ouhai, Wenzhou, Zhejiang 325015, P.R. China E-mail: yukang62@126.com

Dr Shenghui Zhang, Division of Clinical Research, The First Affiliated Hospital of Wenzhou Medical University, Shangcai Village, Nanbaixiang Town, Ouhai, Wenzhou, Zhejiang 325015, P.R. China E-mail: shenghuizhang1@126.com

*Contributed equally

Key words: casein kinase $1 \alpha$, acute myeloid leukemia, autophagy, apoptosis, p53 phosphorylation of mammalian target of rapamycin (mTOR). Our findings indicate that CK1 $\alpha$ promotes AML by suppressing p53 downstream of MDM2-mediated autophagy and apoptosis, suggesting that targeting CK1 $\alpha$ provides a therapeutic opportunity to treat AML.

\section{Introduction}

Acute myeloid leukemia (AML) is a genetically heterogeneous disease characterized by infiltration of the bone marrow (BM), blood, and other tissues with proliferative, clonal, abnormally differentiated cells of the hematopoietic origin (1). Although the overall survival rate of AML has increased significantly, relapse after remission is still the most important issue for AML treatment. Mounting evidence shows that leukemia stem cells (LSCs) can initiate tumor formation and lead to chemotherapy failure or resistance and disease relapse (2,3). LSCs and leukemic blasts ensure their survival by utilizing autophagy to respond to the specific metabolic demands during rapid cell proliferation and to counteract chemotherapeutic stress (4).

Autophagy is an evolutionarily conserved process during which intracellular components are catabolized to sustain energy metabolism homeostasis and to safeguard cells against stress (5). There are many reports concerning the function of autophagy in AML. Usually, we consider autophagy as a protective mechanism in AML. Larrue et al (6) reported that bortezomib could induce FLT3-ITD degradation through autophagy which resulted in the death of AML cells. Jin et al (7) believed that granulocytic AML differentiation relies on noncanonical autophagy pathways and that restoring autophagic activity may be beneficial in differentiation therapies. Several studies have shown that low level of basal autophagy gene expression such as ATG5, ATG7 and LC3 $(7,8)$, and reduced autophagic flux occur in patient-derived AML blasts, and the loss of core autophagy genes results in leukemia initiation and progression in mouse models (9). Pharmacological or genetic inhibition of autophagy leads to impaired leukemic cell viability and increased sensitivity to standard chemotherapy $(6,7,10,11)$.

$\beta$-catenin has been demonstrated as a critical regulator of the self-renewal and therapeutic target for various cancer stem cells including leukemia-initiating cells (LICs) $(12,13)$. Casein kinase $1 \alpha(C K 1 \alpha)$, encoded by $C S N K 1 A 1$, is a classical negative regulator 
for the Wnt $/ \beta$-catenin signaling pathway (14). In addition to Wnt $/ \beta$-catenin signaling, $\mathrm{CK} 1 \alpha$ also regulates p53, GLI transcription factors and other important signaling pathways (15-17). $\mathrm{CK} 1 \alpha$ has been reported to be implicated in apoptosis, survival, senescence and other cellular physiological processes, thus regulating the occurrence and development of multiple tumors $(17,18)$. Recently, CK1 $\alpha$ was reported to act as a key negative regulator of oncogenic RAS-induced autophagy, suggesting that targeting $\mathrm{CK} 1 \alpha$-regulated autophagy provides a promising therapeutic opportunity to treat oncogenic RAS-driven cancers (19). In contrast to solid tumors, the role of $\mathrm{CK} 1 \alpha$ in regulating autophagy in hematologic malignancies is less well defined.

In the present study, we found that $\mathrm{CK} 1 \alpha$ was upregulated and correlated with prognosis in AML. Furthermore, CK1 $\alpha$ was found to inhibit p53 downstream of murine double minute 2 (MDM2)-mediated autophagy and apoptosis. Pharmacological or genetic inhibition of CK1 $\alpha$ suppressed the proliferation and increased the autophagy flux as well as apoptosis in multiple AML cell lines and patient blast cells. Spautin-1, a specific autophagy inhibitor (20), aggravated the cell death induced by D4476, a classical inhibitor of CK1 $\alpha$ (21), suggesting that CK1 $\alpha$ inhibition-mediated autophagy might be a pro-survival signaling in AML. Additionally, D4476 upregulated p53 and phosphorylated 5' AMP-activated protein kinase (AMPK), and reduced the phosphorylation of mammalian target of rapamycin (mTOR).

\section{Materials and methods}

AML cell lines and primary cell separation. Human AML cell lines HL-60, HEL and THP-1 were purchased from the Cell Bank of the Chinese Academy of Sciences (Shanghai, China), and cultured in RPMI-1640 medium supplemented with $7 \%$ fetal bovine serum (FBS; both from Gibco; Thermo Fisher Scientific, Inc.) at $37^{\circ} \mathrm{C}$ in $5 \% \mathrm{CO}_{2}$. A total of 61 newly diagnosed AML patients and 6 AML patients in complete remission (CR) in the First Affiliated Hospital of Wenzhou Medical University from March to December 2018 according to the FAB classification system were enrolled in this study (Table SI). Eleven age-matched healthy donors were enrolled as controls. Bone marrow mononuclear cells (BMMNCs) were isolated using Ficoll-Hypaque (Haoyang Institute of Biotechnology, China). In addition, BMMNCs of the newly diagnosed AML patients were not used for the following experiments until the percentage of $\mathrm{CD} 45^{\mathrm{dim}} \mathrm{SSC}^{\mathrm{dim}}$ cells was more than $70 \%$ using flow cytometric analyses. All primary blast cells were cultured in RPMI-1640 medium supplemented with $20 \% \mathrm{FBS}$ at $37^{\circ} \mathrm{C}$ in $5 \% \mathrm{CO}_{2}$.

This study was approved by the Institutional Review Board of the First Affiliated Hospital of Wenzhou Medical University, and informed consent was obtained from all participants in accordance with the Declaration of Helsinki protocol.

TCGA data analysis. The relationship of CSNK1A1 expression and the prognosis in patients with AML was analyzed using UALCAN (22), an interactive web-portal to perform to in-depth analyses of TCGA gene expression data. UALCAN is publicly available at http://ualcan.path.uab.edu.

Quantitative real-time PCR. The total RNA was extracted from cells using Trizol reagent (Life Technologies, USA) and reverse transcribed into cDNA with following procedure: $42^{\circ} \mathrm{C}$ for $30 \mathrm{~min}, 70^{\circ} \mathrm{C}$ for $15 \mathrm{~min}$, and then cDNA was amplified and quantified according to the procedure: $95^{\circ} \mathrm{C}$ for $30 \mathrm{sec}$, followed by 40 cycles at $95^{\circ} \mathrm{C}$ for $5 \mathrm{sec}$ and at $60^{\circ} \mathrm{C}$ for $34 \mathrm{sec}$ by ABI Prism 7500 using SYBR Green PCR Master Mix (Takara) with primer pairs. All results were normalized against GAPDH, RQ=2- $2^{-\Delta \mathrm{Cq}}(23)$. Primer sequences are listed in Table SII.

Lentiviral-mediated RNA interference. Four separate short hairpin RNA (shRNA) constructs targeting CSNK1Al

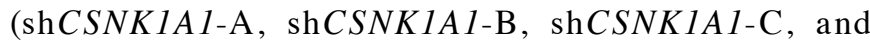
shCSNK1A1-D) and one control shRNA construct targeting lactose gene were synthesized (Hanyin Biotechnology Co., Ltd.). The sequences of the target sequences for shRNAs and synthetic oligo information are shown in Table SIII. Each sequence pair was cloned into the LV3 shuttle plasmid with a RSV and CMV and H1 three promoter-driven GFP expression cassette, respectively. Each lentiviral expression plasmid and three corresponding packaging plasmids were co-transfected into 293T cells with RNAi-Mate reagent (GenePharma), respectively. Supernatants were collected $72 \mathrm{~h}$ after transfection, and the final virus titer in the supernatant was $1.3 \times 10^{9} \mathrm{TU} / \mathrm{ml}$ for each lentivirus. AML cells were infected with the lentivirus in the presence of $10 \mu \mathrm{g} / \mathrm{ml}$ polybrene, and $72 \mathrm{~h}$ after infection, $3 \mu \mathrm{g} / \mathrm{ml}$ puromycin was used to screen those successfully transfected cells. The efficiency of gene silencing was examined using RT-PCR and western blot analysis.

Western blot analysis and co-immunoprecipitation (Co-IP). The cells were harvested, and lysed with RIPA lysis buffer (Beyotime) containing PMSF and protease inhibitor cocktail (Beyotime). The protein was boiled and subjected to western blot analysis with different antibodies, including p53 [Cell Signaling Technology, Inc. (CST), \#2527], SQSTM1/p62 (CST, \#8025), p-AMPK (CST, \#4184), AMPK (CST, \#2532), mTOR (CST, \#4517), p-mTOR (CST, \#5536), PARP (CST, \#9542), LC3-I/II (CST, \#4108), GAPDH (CST, \#2118), CK1 $\alpha$ (Abcam, ab206652), ATG-7 (CST, \#2631), at 1/1,000 dilution for incubation overnight at $4^{\circ} \mathrm{C}$, respectively. For Co-IP, the cells were lysed with RIPA lysis buffer containing PMSF on ice for $0.5 \mathrm{~h}$. Protein concentration was determined using a BCA protein assay kit. Five hundred micrograms of protein per sample was used and coated with $20 \mu \mathrm{l}$ protein A/G-agarose beads and $1 \mu \mathrm{g}$ mouse-control $\mathrm{IgG}$ with protein to eliminate non-specific proteins for $1 \mathrm{~h}$, and then was centrifugated at $2,400 \times \mathrm{g}$ for $5 \mathrm{~min}$. The supernatants were incubated with beads coated with $10 \mu \mathrm{g} / \mathrm{ml}$ mouse-source anti-MDM2 or $1 \mu \mathrm{g}$ control $\mathrm{IgG}$ overnight at $4^{\circ} \mathrm{C}$. Beads were washed three times with lysis buffer and boiled for $5 \mathrm{~min}$. The bead-protein mixture was subjected to western blotting with antibodies against mouse-source anti-MDM2 (\#AF208, Affinity, at 1/1,000 dilution), rabbit-source anti-p53 (\#2527, CST, at 1/1,000 dilution), anti-CK1 $\alpha$ (ab206652, Abcam, at 1/1,000 dilution), respectively. Optical densities of the bands were scanned and analyzed with FluorChem E and AlphaView SA software (Version: 3.4.0.0) (both from ProteinSimple).

Flow cytometry assay and cell viability assay. Apoptosis assay was measured using the Annexin V-FITC/PI apoptosis kit and 

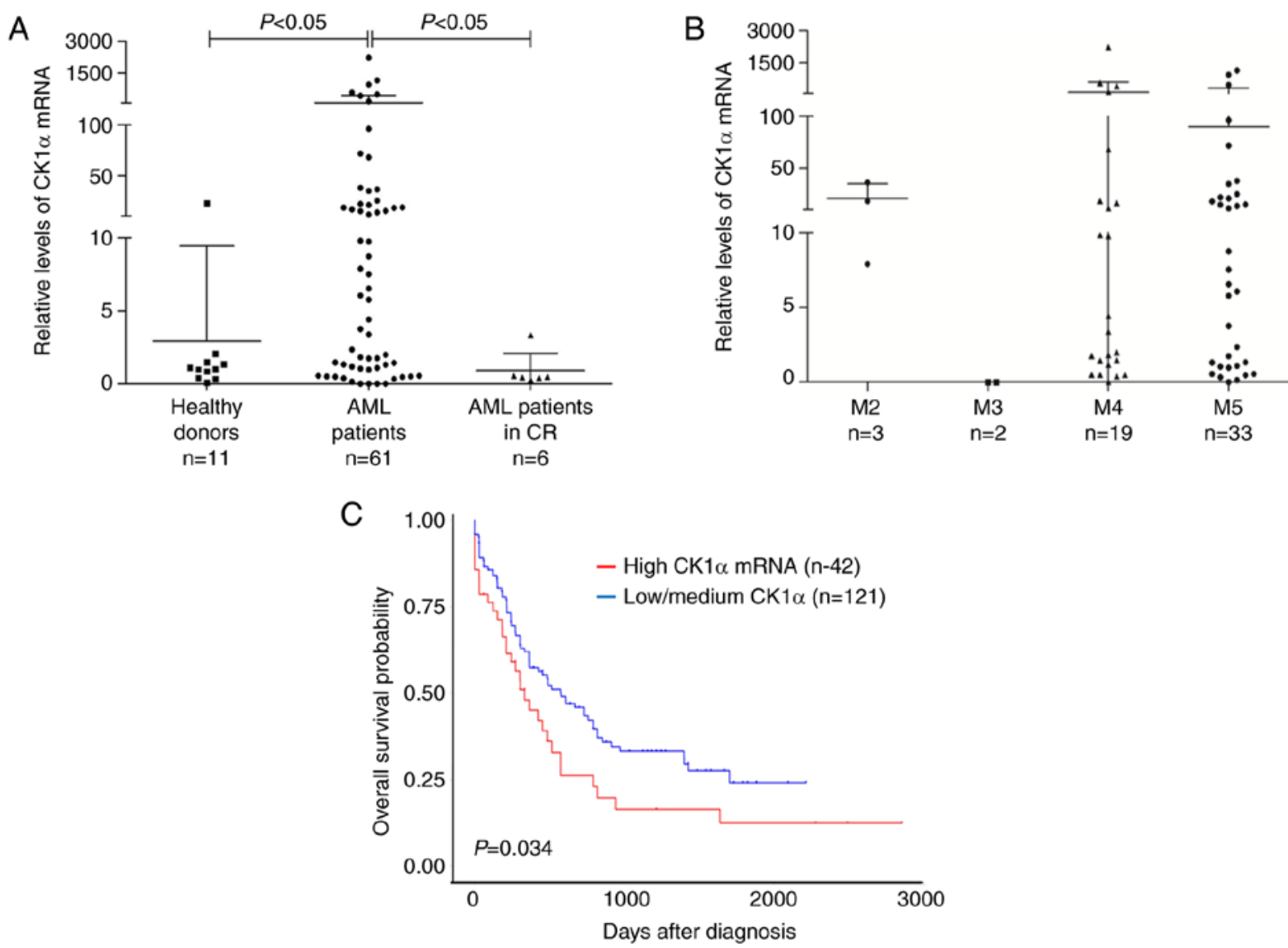

Figure 1. The level of CK1 $\alpha$ mRNA is highly expressed and correlated with poor prognosis in patients with AML. (A) Bone marrow specimens from 67 patients with AML (61 newly diagnosed, 6 achieved CR) and 11 healthy donors were collected. The level of CK1 $\alpha$ mRNA was determined by RT-qPCR and GAPDH was used as an internal reference. (B) The level of CK1 $\alpha$ mRNA was showed in each FAB subtypes of 61 newly diagnosed patients with AML. (C) Kaplan-Meier survival analysis was performed based on the survival data of 163 AML patients and its CSNK1A1 expression information in the TCGA-LAML database using the UALCAN website. The upper quartile of the expression information was used as a boundary value and differences in survival distributions were evaluated by the log-rank test. CK1 $\alpha$, casein kinase $1 \alpha$; AML, acute myeloid leukemia; TCGA, Cancer Genome Atlas.

Annexin V-APC/7-AAD apoptosis kit (MultiSciences Biotech Co., Ltd.) as described previously (24). The cell viability assay was measured using the Cell Counting Kit-8 (CCK-8, Dojindo, Japan) according to the manufacturer's protocol.

Colony formation assay. The cells were cultured in methylcellulose medium (MethoCult ${ }^{\mathrm{TM}} \mathrm{H} 4034$, Stemcell Technologies) according to the manufacturer's protocol. Visible colonies, defined to consist of at least 60 cells were counted. The colonies were photographed by microscope (BX51, Olympus, Japan) and the total cells were counted after rinsing with PBS.

Transmission electron microscope (TEM). Cell masses were fixed with $2.5 \%$ glutaraldehyde and were cut into $1 \times 1 \times 1 \mathrm{~mm}$ pieces. Then, the cell masses were cleaned by $0.1 \mathrm{M}$ phosphoric acid bleach, dehydrated by 50,70,90 and 100\% ethanol and $100 \%$ epoxy for $15 \mathrm{~min}$, embedded with epoxypropane + buried solution $(2: 1)$ for $2 \mathrm{~h}$, epoxypropane + burden (1:2) for $3 \mathrm{~h}$, pure buried liquid overnight and pure buried liquid for $4 \mathrm{~h}$, sliced by slim slicer $(70 \mathrm{~nm})$, and stained by 3\% uranium acetate-lead citrate. Finally, the mitochondrial membrane and typical autophagic vacuoles of each cell sample were observed under $\mathrm{x} 20,000$ magnification using a transmission electron microscope (Jeol JEM 1230, Japan).
Statistical analysis. Two-tailed unpaired Student's t-tests with $95 \%$ confidence interval (CI) were used to analyze the data involving direct comparison of an experimental group with a control group. One-way ANOVA tests with Dunnett's method for multiple comparisons with $95 \%$ CI were used to analyze the data involving 2 or more test groups and a control group. One-way ANOVA with Tukey test was used to perform multiple comparisons for up to 3 groups. P-value $<0.05$ was considered to indicate a statistically significant difference. All experiments were performed with three replicates, unless stated.

\section{Results}

CKla is overexpressed and its inhibition impairs the proliferation and the colony formation in AML cells. Mounting evidence indicates that $\beta$-catenin is overexpressed and promotes disease recurrence contributing to a poor prognosis in AML (25), which motivated us to investigate whether CK1 $\alpha$, a negative regulator of $\beta$-catenin, also has a similar role. CK1 $\alpha$ mRNA was significantly higher in BMMNCs from the newly diagnosed AML patients than those from healthy donors or AML patients who achieved CR (Fig. 1A). There was no difference in the level of CK1 $1 \alpha$ mRNA among the different types 


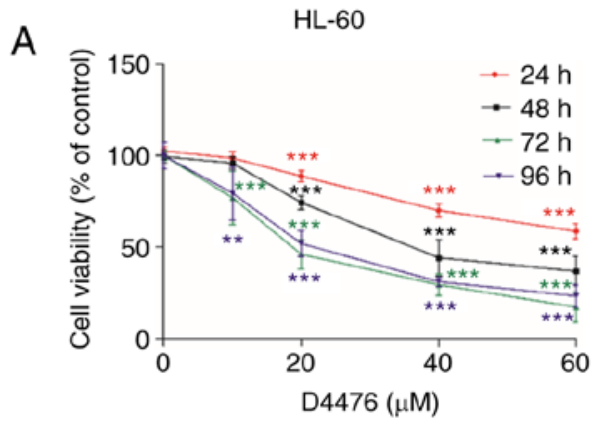

THP-1

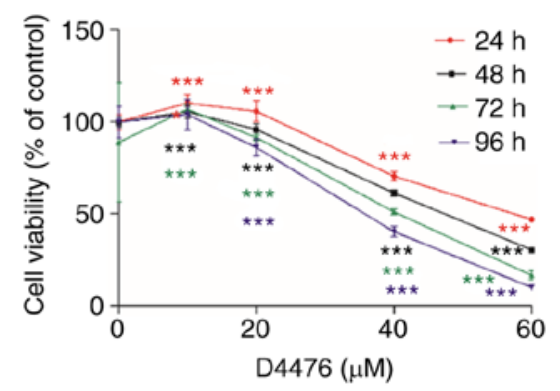

HEL



Primary blast cells

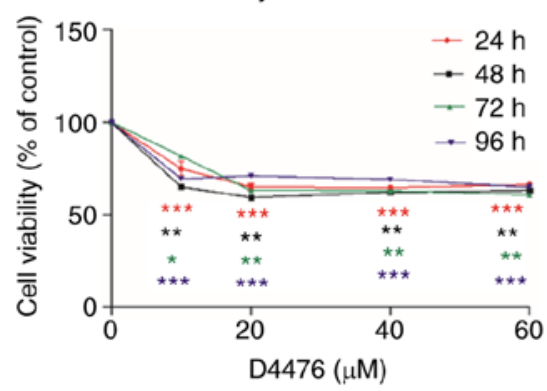

B Control

$40 \mu \mathrm{M}$ D4476
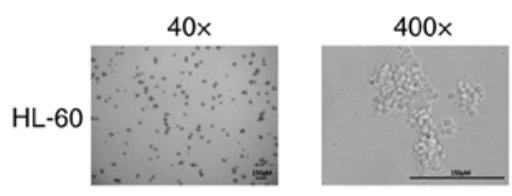

HEL
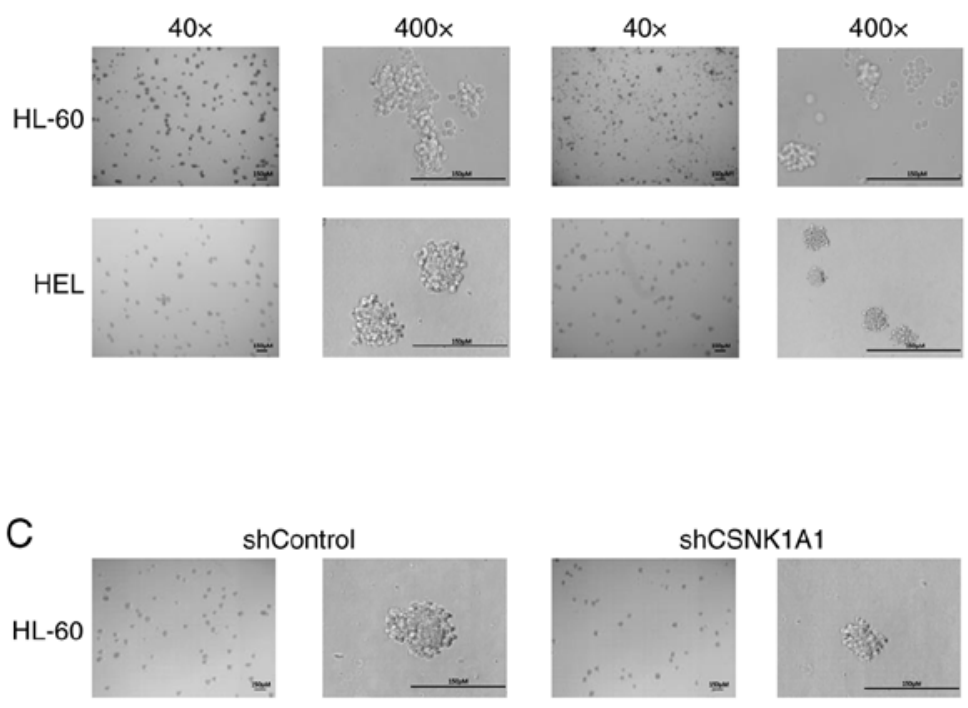

shControl
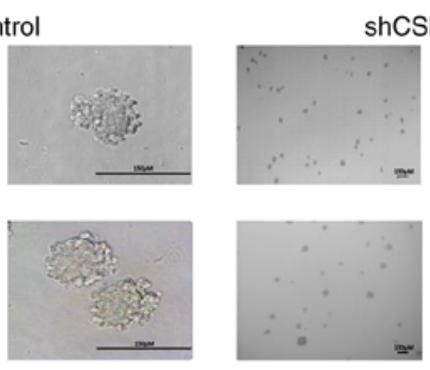

shCSNK1A

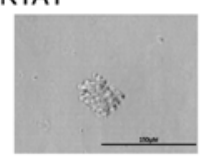

HEL
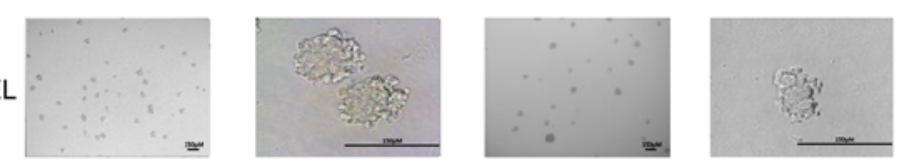
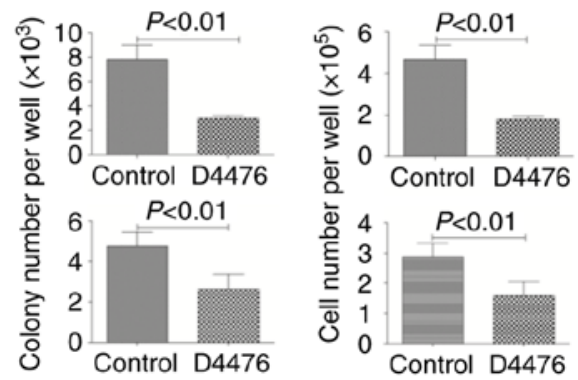

¿ $4 \quad P<0.01$
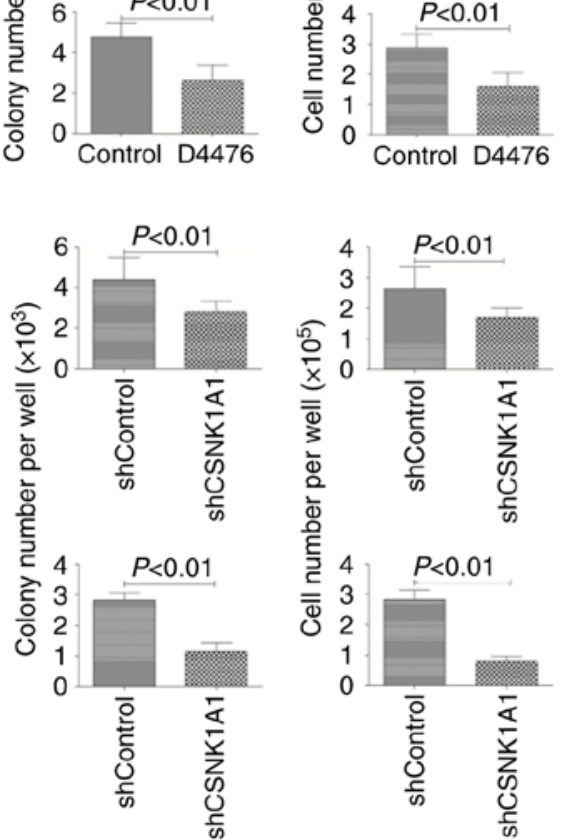

Figure 2. Inhibition of CK1 $\alpha$ using D4476 or lentivirus-mediated shRNA impairs cell survival in AML cells. (A) The cell viability was determined by CCK-8 assay in three AML cell lines HL-60, HEL, and THP-1 cells as well as in patient blast cells after treatment with various concentrations (0-60 $\mu \mathrm{M})$ of D4476 for $24,48,72$ and $96 \mathrm{~h}$. Statistical analysis was performed using Dunnett's $t$ test; ${ }^{*} \mathrm{P}<0.05,{ }^{* *} \mathrm{P}<0.01$ and ${ }^{* * *} \mathrm{P}<0.001$. (B and C) The morphological changes in cell colonies were observed in HL-60 and HEL cells with or without $40 \mu \mathrm{M}$ D4476, or with shControl or shCSNK1A1 for 8 days. Representative images (left panel) are shown and quantitative data of colony and cell number (right panel) representing four independent experiments are presented in bar charts. CK1 $\alpha$, casein kinase $1 \alpha$; AML, acute myeloid leukemia.

of FAB (Fig. 1B). Furthermore, the patients with a high level of CK1 $\alpha$ mRNA showed a poorer outcome than those with a low or medium level of CK1 $\alpha$ mRNA (P=0.034, Fig. 1C).
These findings suggested that CK1 $\alpha$ may be overexpressed and confer a poor prognosis in AML. All patient information is listed in Table SI. 

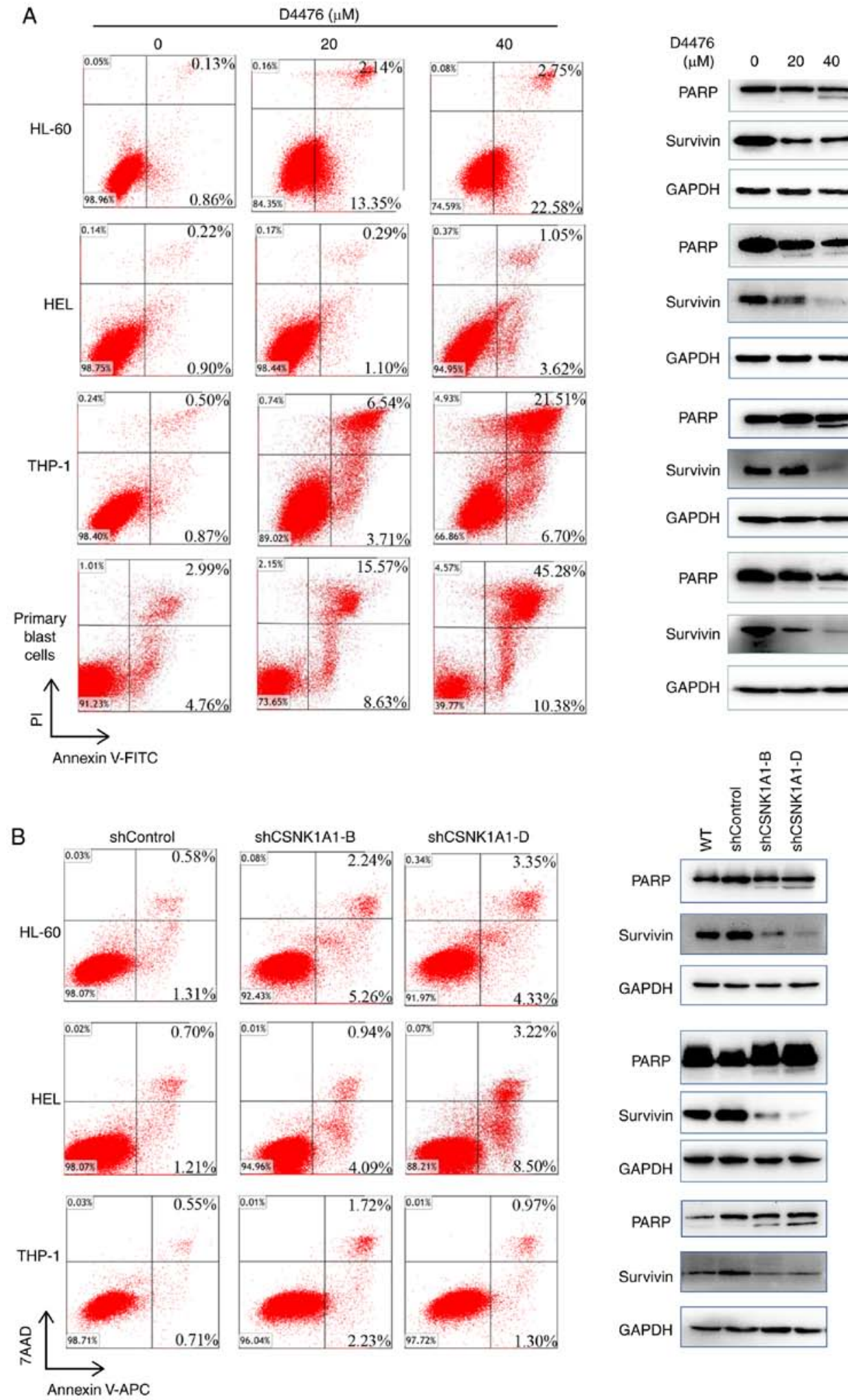

Figure 3. Inhibition of CK1 $\alpha$ induces apoptosis in AML cells. (A) Three AML cell lines HL-60, THP-1 and HEL as well as patient blast cells were treated with 0, 20, $40 \mu \mathrm{M} \mathrm{D} 4476$ for $48 \mathrm{~h}$, and the apoptosis was determined by Annexin V-FITC/PI. Meanwhile, the cleavage of PARP and the expression of anti-apoptotic protein survivin were also assessed. Images representing four independent experiment are shown. (B) HL-60, THP-1 and HEL cells were treated with lentivirus-mediated shRNA for $72 \mathrm{~h}$, and the apoptosis was subsequently determined using Annexin V-APC/7AAD. The cleavage of PARP and the expression of survivin were also determined. Images representing four independent experiment are shown. CK1 $\alpha$, casein kinase 1 $\alpha$; AML, acute myeloid leukemia; PARP, poly(ADP-ribose) polymerase.

To investigate the role of CK1 $\alpha$ in AML cells, we decreased CK1 $\alpha$ using pharmacological or genetic inhibition. We found that D4476 significantly reduced the cell viability of three AML cell lines HL-60, HEL and THP-1 in a time- and dose-dependent manner (Fig. 2A), while only a dose-dependent manner was shown after treatment with D4476 lower than 
$10 \mu \mathrm{M}$ in patient blast cells. We further investigated whether D4476 affects the colony formation ability of AML cells, and found that D4476 at $40 \mu \mathrm{M}$ dramatically impaired the colony formation, as indicated with colony number and total cell number, in both HL-60 and HEL cells (Fig. 2B). Notably, there were many small cell clusters, but did not reach the standard of a colony in the D4476 treatment group in these two cell lines (Fig. 2B). Meanwhile, similar results were also observed in these two AML cell lines with genetic inhibition of CK1 $\alpha$ using lentiviral-mediated shRNA (Figs. 2C and S1). Importantly, we found that the colony size was decreased in both the D4476 treatment group and the shCSNK1Al group (Fig. 2B and C), suggesting that pharmacological or genetic inhibition of $\mathrm{CK} 1 \alpha$ affects the colony formation mainly through decreasing the size of the colony in AML cells.

CK1 $\alpha$ inhibition induces apoptosis but does not influence cell cycle in the AML cells. We found that D4476 dramatically induced apoptosis in a dose-dependent manner in three AML cell lines tested as well as patient blast cells (Fig. 3A). As expected, D4476 treatment promoted the cleavage of PARP, a classical marker of apoptosis, and decreased the expression of survivin, a negative marker protein of apoptosis, in HL-60, THP-1 and HEL cells as well as in patient blast cells (Fig. 3A). Similarly, the genetic inhibition of $\mathrm{CK} 1 \alpha$ also induced a moderate apoptosis in the three AML cell lines HL-60, THP-1 and HEL (Fig. 3B). Furthermore, combination with cytarabine (Ara-c) and D4476 or gene silencing of CK1 $\alpha$ had a synergistic effect on the induction of apoptosis in THP-1 and HEL cells (Fig. S2). In addition, we found that pharmacological or genetic inhibition of $\mathrm{CK} 1 \alpha$ had almost no effect on cell cycle distribution of HL-60 or HEL cells (Fig. S3).

CK1 $\alpha$ inhibition promotes autophagy in AML cells. Autophagy is a cellular process activated in response to various forms of stresses such as nutrient deprivation, hypoxia and endoplasmic reticulum (ER) stress. Apoptosis triggered by ER stress is often accompanied by increased autophagy. Apoptosis induced by $\mathrm{CK} 1 \alpha$ inhibition prompted us to investigate whether CK $1 \alpha$ inhibition also activates autophagy. We used TEM to observe autophagosomes and found that pharmacological or genetic inhibition of CK1 $\alpha$ promoted the autophagy flux of HL-60 and HEL cells with increased autophagosomes (Fig. 4A). We found that D4476 treatment upregulated the expression of ATG-7 and LC3-II in all three AML cell lines tested as well as patient blast cells (Fig. 4B), which further confirmed the occurrence of autophagy. SQSTM1/p62, a substrate of LC3-II, is usually reduced when autophagy occurs. However, we found that D4476 treatment concomitantly increased the levels of SQSTM1/p62 and LC3-II protein in HL-60 or HEL cells in a dose-dependent manner within $48 \mathrm{~h}$ (Fig. S4). It has been reported that in nutrient-rich conditions, $\beta$-catenin can inhibit autophagy and the generation of SQSTM1/p62 mRNA by regulating TCF4/LEF, but when autophagy occurs, $\beta$-catenin can be degraded by autophagy and p62 becomes derepressed and dramatically increased (26). We therefore determined the mRNA expression of SQSTM1/p62 and found that D4476 treatment significantly increased the level of SQSTM1/p62 mRNA, suggesting that enhanced autophagy reduced the inhibition of $\beta$-catenin on $\mathrm{p} 62$, resulting in greater p62 generation than degradation, ultimately leading to the accumulation of p62 (Fig. 4C). Furthermore, combination with Ara-c and D4476 resulted in the upregulation of autophagic flux in THP-1 and HEL cells (Fig. S2A).

CK1 $\alpha$ inhibition-mediated autophagy exerts protective effects on AML cells. Once confirmed, autophagy and apoptosis occurring together in the D4476-treated AML cells, the underlying relationship aroused our curiosity. Firstly, we combined Spautin-1 with D4476 to unravel the veil of mystery. Spautin-1 aggravated the cell growth inhibition induced by D4476 on all three AML cell lines tested as well as in the patient blast cells (Fig. 5A). Furthermore, we determined the apoptosis when the autophagy was inhibited. Spautin-1 alone did not induce apoptosis in AML cells, and Spautin-1 potently aggravated D4476-induced apoptosis on HEL cells but merely moderately exacerbated D4476-induced apoptosis in HL-60 cells (Fig. 5B). These data suggested that D4476 combined with Spautin-1 exerted a synergistic antitumor effect on AML cells.

CK1 $\alpha$ interacts with MDM2 and mediates autophagy by targeting p53/AMPK/mTOR signaling pathway in AML. A number of signaling pathways including PI3K/Akt/mTOR, $\mathrm{p} 53 / \mathrm{AMPK} / \mathrm{mTOR}$, and MAPKs, are implicated in autophagic cell survival and cell death $(27,28)$. We found that CK1 $\alpha$ had a relation with MDM2 and p53 (Fig. 5C). It has been recognized that MDM2 is a negative regulator of p53 $(29,30)$ and p53 regulates AMPK/mTOR signaling to activate autophagy (31). We found that CK1 $\alpha$ inhibition by D4476 increased the expression of $\mathrm{p} 53$ and MDM2, promoted the phosphorylation of AMPK, and inhibited the phosphorylation of mTOR in a dose-dependent manner in HL-60 and HEL cells (Fig. 5D). Furthermore, we found that D4476 did not affect the level of MDM2 when CK1 $\alpha$ was genetically inhibited in HEL cells (Fig. 5E), indicating that D4476 may influence MDM2 through $\mathrm{CK} 1 \alpha$. These data suggested that $\mathrm{CK} 1 \alpha$ may regulate $\mathrm{p} 53$ through MDM2, and inhibit autophagy by the AMPK/mTOR signaling pathway downstream of p53.

\section{Discussion}

Casein kinase $1 \alpha(\mathrm{CK} 1 \alpha)$ is able to regulate several important signaling molecules in different types of tumors (32). Especially its ability to regulate the Wnt signaling, p53, and apoptosis induction makes it a potential target in tumor therapy $(32,33)$. In the present study, we found that CK1 $\alpha$ was overexpressed in newly diagnosed acute myeloid leukemia (AML) patient samples, which further confirmed the concept of overexpression of CK1 $\alpha$ in AML based on its expression in multiple AML cell lines detected by gene chip (34). When AML patients achieve complete remission (CR), the level of CK1 $\alpha$ was decreased. Then we analyzed the data of TCGA via UALCAN and found that AML patients with high expression of CK $1 \alpha$ mRNA had poor prognosis compared with those with low or medium expression of CK1 $\alpha$ mRNA. This finding was in accordance with the role of CK1 $\alpha$ in colorectal cancer (35).

The relationship between autophagy and tumors is of current research interest. To the best of our knowledge, this is the first study to reveal the relationship of CK1 $\alpha$ and autophagy in AML. Pharmacological or genetic inhibition of CK $1 \alpha$ induced apoptosis and autophagy in AML cells, which were 

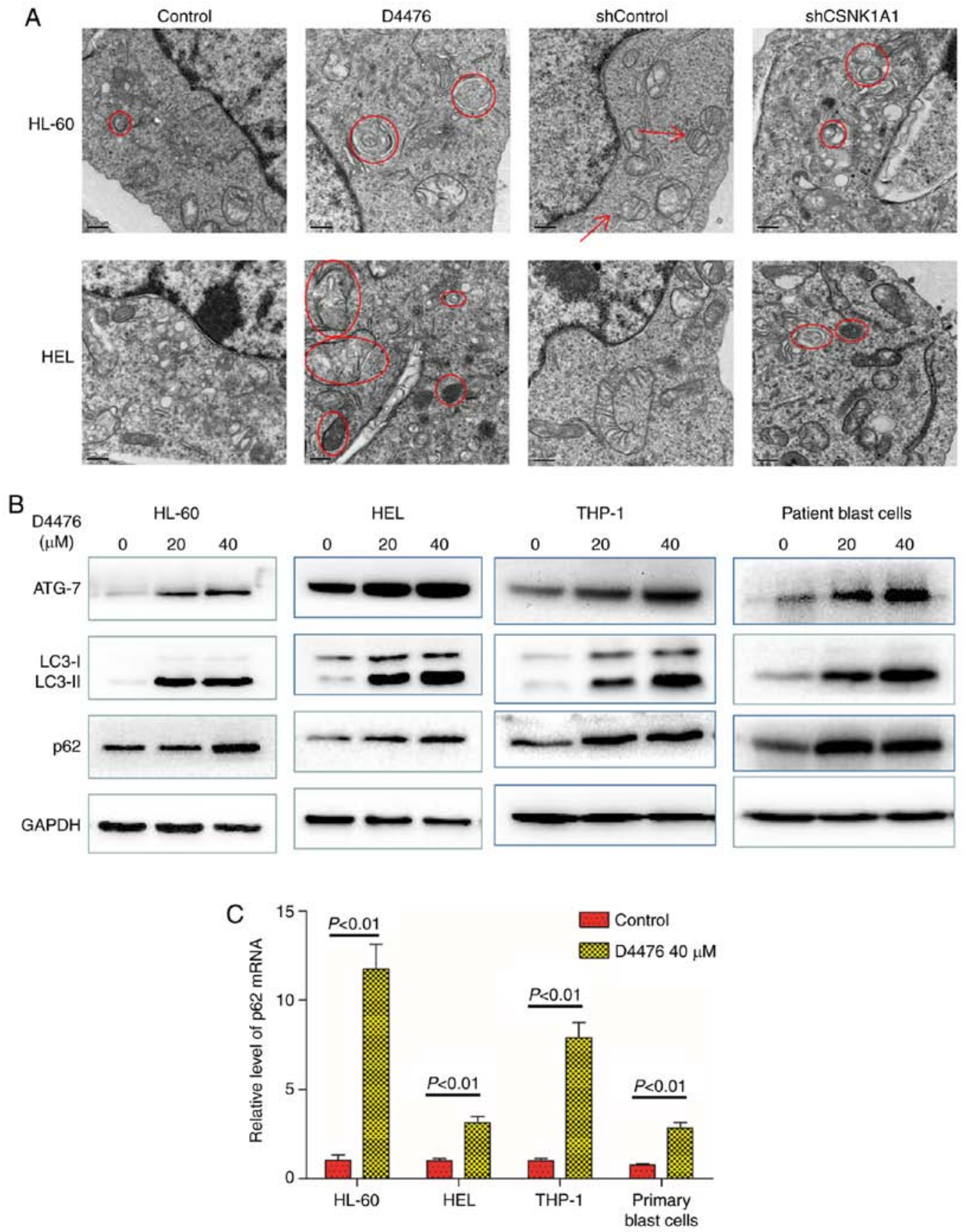

Figure 4. Inhibition of CK1 $\alpha$ promotes autophagy in AML cells. (A) HL-60 and HEL cells were treated with or without $40 \mu \mathrm{M}$ D4476 for $48 \mathrm{~h}$, or transfected with shControl or shCSNK1A1 for $72 \mathrm{~h}$. The cell masses were cleaned, dehydrated, embedded, sliced, stained, and subsequently observed using a transmission electron microscope. Images representing at least 10 views of each group are shown. The red arrows indicate mitochondria, and the red circles indicate autophagosomes. (B) Three AML cell lines (HL-60, HEL, and THP-1) as well as patient blast cells were treated with or without $40 \mu \mathrm{M} \mathrm{D} 4476$ for $48 \mathrm{~h}$ and the autophagy marker proteins p62, ATG-7 and LC3-I/II were determined. Images representing at least 3 independent experiment are shown. (C) The AML cell lines (THP-1, HL-60, and HEL) as well as patient blast cells were treated with or without $40 \mu \mathrm{M}$ D4476 for $48 \mathrm{~h}$ and the level of p62 mRNA was determined by RT-qPCR. Results are expressed as mean $\pm \mathrm{SD}$, representing at least three independent experiments. CK1 $\alpha$, casein kinase $1 \alpha$; AML, acute myeloid leukemia; ATG-7, autophagy related 7; LC3, microtubule-associated protein 1A/1B-light chain 3.

not completely consistent with other cancers, such as multiple myeloma (36). Autophagy is responsible for the degradation of p62 and p62 knockout cells possess higher basal levels of LC3-II, followed by an increase in autophagic function (37). However, in our present study, inhibition of CK1 $\alpha$ induced autophagy and increased p62 mRNA and protein. When CK1 $\alpha$ was inhibited by D4476, autophagy occurred and the inhibitory function via $\beta$-catenin regulating-TCF4 on p62 was attenuated, p62 became derepressed and dramatically increased [21]. We also found that inhibition of CK1 $\alpha$ did not affect cell cycle progression in AML cells, inconsistent with the results of a previous study (32), suggesting that the role of CK1 $\alpha$ on cell cycle progression is cell type-specific.

The relationship between autophagy and apoptosis is two-sided. In some situations, autophagy weakens the apoptotic effect and acts as a pro-survival role (38), but in other situations this is absolutely opposed (24). In the present study, pharmacological inhibition of $\mathrm{CK} 1 \alpha$ by $\mathrm{D} 4476$ induced 

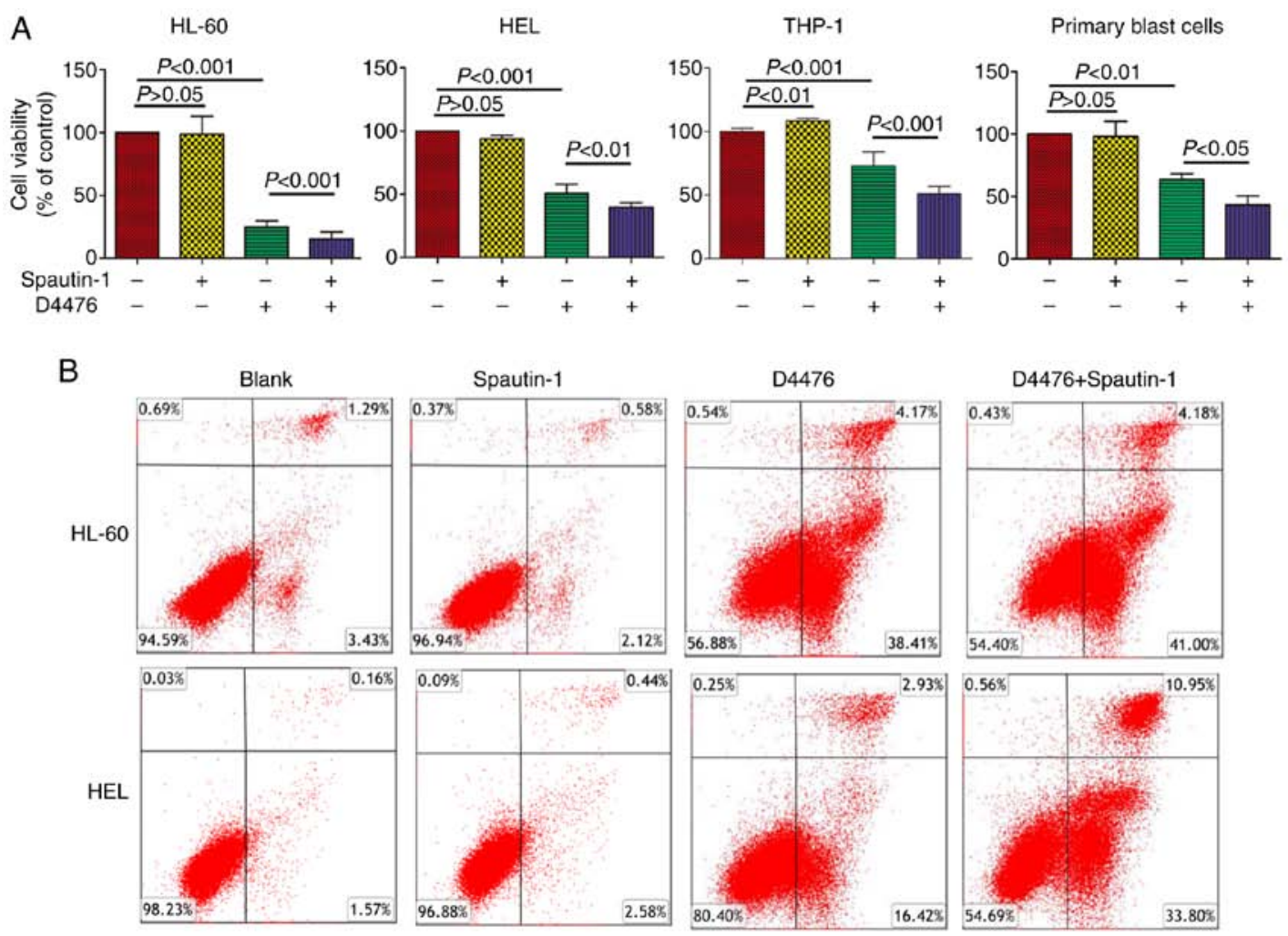

C
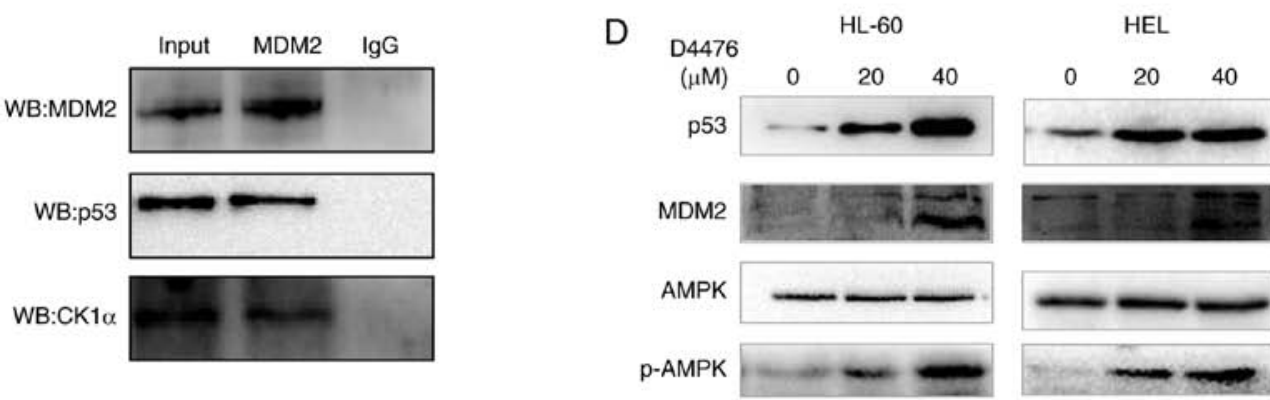

E
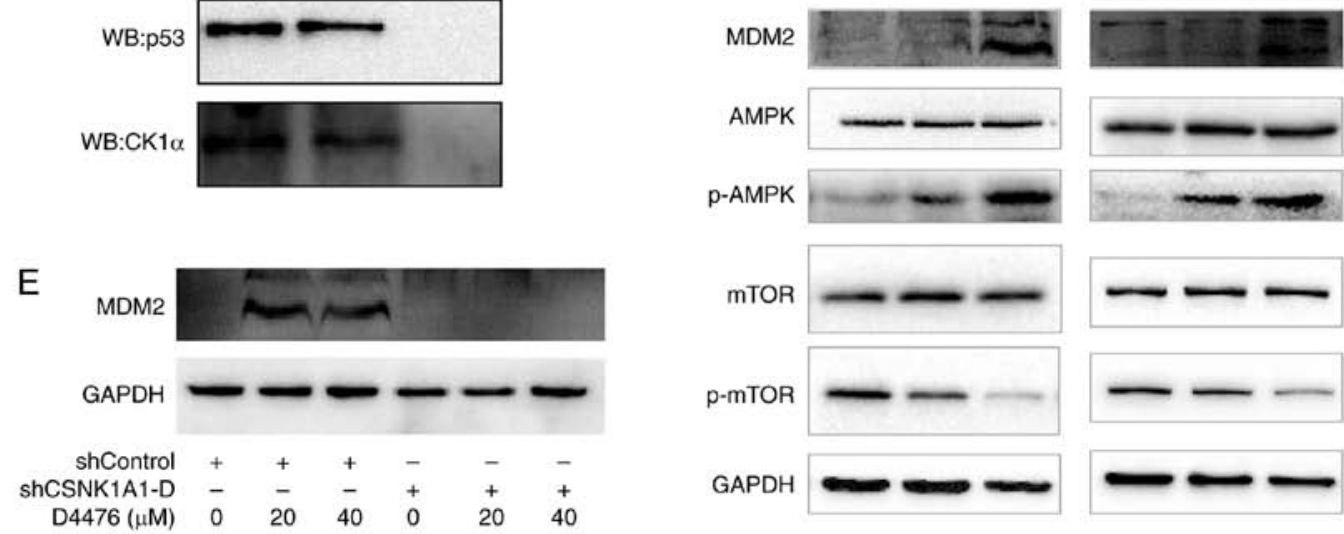

Figure 5. Autophagy inhibitor Spautin-1 aggravates apoptosis induced by D4476, and CK1 $\alpha$ inhibits autophagy by targeting the p53/AMPK/mTOR signaling pathway downstream of MDM2 in AML cells. (A) After treatment with $40 \mu \mathrm{M}$ D4476 in the presence or absence of $10 \mu \mathrm{M}$ autophagy inhibitor Spautin-1 for $48 \mathrm{~h}$, the cell viability was measured by CCK- 8 assay. Data are expressed as mean \pm SD of at least three independent experiments, and statistical analysis was performed by one-way ANOVA with Tukey test. (B) After treatment with $40 \mu \mathrm{M}$ D4476 in the presence or absence of $10 \mu \mathrm{M}$ Spautin-1 for $48 \mathrm{~h}$, apoptosis was determined by Annexin V-FITC/PI. (C) Co-immunoprecipitation showed that CK1 $\alpha$ interacted with MDM2 and p53 in HEL cells. Ten percent whole cell lysate was used as input samples. (D) Treatment with D4476 upregulated the level of p53 and MDM2 and phosphorylated AMPK, and inhibited the phosphorylation of mTOR in both HL-60 and HEL cells. (E) Treatment with D4476 did not significantly alter the level of MDM2 when CK1 $\alpha$ was genetically inhibited in HEL cells. Images representing at least three independent experiments are shown. CK1 $\alpha$, casein kinase 1 $\alpha$; AML, acute myeloid leukemia; MDM2, murine double minute 2; mTOR, mammalian target of rapamycin; AMPK, 5' AMP-activated protein kinase.

obvious apoptosis with increased cleavage of PARP and decreased survivin in a dose-dependent manner in AML cells. Furthermore, autophagy inhibitor Spautin-1 exacerbated D4476-mediated apoptosis, suggesting that CK1 $\alpha$ inhibition-mediated autophagy may be a pro-survival element, and co-targeting CK1 $\alpha$ and autophagy may confer a more effective anti-leukemia effect.
Murine double minute 2 (MDM2), a negative regulator of the tumor-suppressor p53, functions as an inhibitor of apoptosis in AML (39). CK1 $\alpha$ plays a central role in mediating MDM2 control of p53 protein stability (40). Inhibition of CK1 $\alpha$ has p53-dependent therapeutic efficacy in AML (32). The activation of p53 and the AMPK/mTOR signaling pathway (27) plays a critical role in regulating autophagy. In our present study, 
CK1 $\alpha$ inhibitor D4476 upregulated the levels of MDM2 and p53 in AML cells, similarly with the results of melanoma cell line A375 as described previously (41). In normal conditions, CK1 $\alpha$ promotes p53 degradation and inhibition by MDM2. Therefore, when CK1 $\alpha$ was inhibited by D4476, the complex of $\mathrm{CK} 1 \alpha$ and MDM2 was disrupted, which subsequently resulted in the upregulation of $\mathrm{p} 53$. It has been reported that p53 can bind the MDM2 P2 promoter and transcriptionally upregulate MDM2 expression (32). D4476 did not increase the level of MDM2 when CK1 $\alpha$ was genetically inhibited in HEL cells, indicating that $\mathrm{CK} 1 \alpha$ may influence p53 through MDM2.

In conclusion, our findings demonstrated that CK $1 \alpha$ is overexpressed in AML patients and acts as a negative element in the prognosis of AML patients. CK1 $\alpha$ inhibits p53 downstream of MDM2-mediated autophagy and apoptosis, and the targeting of $\mathrm{CK} 1 \alpha$ and autophagy may offer a potential therapeutic opportunity to treat AML.

\section{Acknowledgements}

Not applicable.

\section{Funding}

This research was supported by the Zhejiang Provincial Natural Science Foundation of China (no. LY20H080005), and the Health Commission of Zhejiang Province (no. 2020KY179).

\section{Availability of data and materials}

The datasets used and/or analyzed during the present study are available from the corresponding author on reasonable request.

\section{Authors' contributions}

SZ and KY designed the research. WX, YG, ZH, RC, YH, and BX performed the experiments. WX, SJ, ZY, SZ and KY analyzed the data; and WX and SZ wrote the paper. All authors read and approved the manuscript and agree to be accountable for all aspects of the research in ensuring that the accuracy or integrity of any part of the work are appropriately investigated and resolved.

\section{Ethics approval and consent to participate}

This study was approved by the Institutional Review Board of the First Affiliated Hospital of Wenzhou Medical University (Wenzhou, China), and informed consent was obtained from all participants in accordance with the Declaration of Helsinki protocol.

\section{Patient consent for publication}

No applicable.

\section{Competing interests}

The authors declare that they have no competing interests.

\section{References}

1. Döhner H, Weisdorf DJ and Bloomfield CD: Acute myeloid leukemia. N Engl J Med 373: 1136-1152, 2015.

2. Thomas D and Majeti R: Biology and relevance of human acute myeloid leukemia stem cells. Blood 129: 1577-1585, 2017.

3. Ho TC, LaMere M, Stevens BM, Ashton JM, Myers JR, O'Dwyer KM, Liesveld JL, Mendler JH, Guzman M, Morrissette JD, et al: Evolution of acute myelogenous leukemia stem cell properties after treatment and progression. Blood 128: 1671-1678, 2016.

4. Rothe K, Porter V and Jiang X: Current outlook on autophagy in human leukemia: Foe in cancer stem cells and drug resistance, friend in new therapeutic interventions. Int J Mol Sci 20: 461, 2019.

5. Hansen M, Rubinsztein DC and Walker DW: Autophagy as a promoter of longevity: Insights from model organisms. Nat Rev Mol Cell Biol 19: 579-593, 2018.

6. Larrue C, Saland E, Boutzen H, Vergez F, David M, Joffre C, Hospital MA, Tamburini J, Delabesse E, Manenti S, et al: Proteasome inhibitors induce FLT3-ITD degradation through autophagy in AML cells. Blood 127: 882-892, 2016.

7. Jin J, Britschgi A, Schläfli AM, Humbert M, Shan-Krauer D, Batliner J, Federzoni EA, Ernst M, Torbett BE, Yousefi S, et al: Low autophagy (ATG) gene expression is associated with an immature AML blast cell phenotype and can be restored during AML differentiation therapy. Oxid Med Cell Longev 2018: $1482795,2018$.

8. Mohamadimaram M, Allahbakhshian Farsani M, Mirzaeian A, Shahsavan S, Hajifathali A, Parkhihdeh S and Mohammadi MH: Evaluation of ATG7 and light chain 3 (LC3) autophagy genes expression in AML patients. Iran J Pharm Res 18: 1060-1066, 2019.

9. Rudat S, Pfaus A, Cheng YY, Holtmann J, Ellegast JM, Bühler C, Marcantonio DD, Martinez E, Göllner S, Wickenhauser C, et al: RET-mediated autophagy suppression as targetable co-dependence in acute myeloid leukemia. Leukemia 32: 2189-2202, 2018.

10. Hu X, Mei S, Meng W, Xue S, Jiang L, Yang Y, Hui L, Chen Y and Guan MX: CXCR4-mediated signaling regulates autophagy and influences acute myeloid leukemia cell survival and drug resistance. Cancer Lett 425: 1-12, 2018.

11. Heydt Q, Larrue C, Saland E, Bertoli S, Sarry JE, Besson A, Manenti S, Joffre C and Mansat-De Mas V: Oncogenic FLT3-ITD supports autophagy via ATF4 in acute myeloid leukemia. Oncogene 37: 787-797, 2018.

12. Siriboonpiputtana T, Zeisig BB, Zarowiecki M, Fung TK, Mallardo M, Tsai CT, Lau PNI, Hoang QC, Veiga P, Barnes J, et al: Transcriptional memory of cells of origin overrides $\beta$-catenin requirement of MLL cancer stem cells. EMBO J 36: 3139-3155, 2017.

13. Zhang Y, Xia F, Liu X, Yu Z, Xie L, Liu L, Chen C, Jiang H, Hao X, He X, et al: JAM3 maintains leukemia-initiating cell self-renewal through LRP5/AKT/ $\beta$-catenin/CCND1 signaling. J Clin Invest 128: 1737-1751, 2018.

14. Thorne CA, Hanson AJ, Schneider J, Tahinci E, Orton D, Cselenyi CS, Jernigan KK, Meyers KC, Hang BI, Waterson AG, et al: Small-molecule inhibition of Wnt signaling through activation of casein kinase $1 \alpha$. Nat Chem Biol 6: 829-836, 2010.

15. Chang CH, Kuo CJ, Ito T, Su YY, Jiang ST, Chiu MH, Lin YH, Nist A, Mernberger M, Stiewe T, et al: CK1 $\alpha$ ablation in keratinocytes induces p53-dependent, sunburn-protective skin hyperpigmentation. Proc Natl Acad Sci USA 114: E8035-E8044, 2017.

16. Elyada E, Pribluda A, Goldstein RE, Morgenstern Y, Brachya G, Cojocaru G, Snir-Alkalay I, Burstain I, Haffner-Krausz R, Jung S, et al: CKI $\alpha$ ablation highlights a critical role for p53 in invasiveness control. Nature 470: 409-413, 2011.

17. Rodriguez-Blanco J, Li B, Long J, Shen C, Yang F, Orton D, Collins S, Kasahara N, Ayad NG, McCrea HJ, et al: A CK1 $\alpha$ activator penetrates the brain and shows efficacy against drug-resistant metastatic medulloblastoma. Clin Cancer Res 25: 1379-1388, 2019.

18. Minzel W, Venkatachalam A, Fink A, Hung E, Brachya G, Burstain I, Shaham M, Rivlin A, Omer I, Zinger A, et al: Small molecules co-targeting CKI $\alpha$ and the transcriptional kinases CDK7/9 control AML in preclinical models. Cell 175: 171-185. e125, 2018.

19. Cheong JK,Zhang F, Chua PJ, Bay BH, Thorburn A and Virshup DM: Casein kinase 1 $\alpha$-dependent feedback loop controls autophagy in RAS-driven cancers. J Clin Invest 125: 1401-1418, 2015. 
20. Liu J, Xia H, Kim M, Xu L, Li Y, Zhang L, Cai Y, Norberg HV, Zhang T, Furuya T, et al: Beclin1 controls the levels of p53 by regulating the deubiquitination activity of USP10 and USP13. Cell 147: 223-234, 2011.

21. Rena G, Bain J, Elliott M and Cohen P: D4476, a cell-permeant inhibitor of CK1, suppresses the site-specific phosphorylation and nuclear exclusion of FOXO1a. EMBO Rep 5: 60-65, 2004.

22. Chandrashekar DS, Bashel B, Balasubramanya SAH, Creighton CJ, Ponce-Rodriguez I, Chakravarthi BVSK and Varambally S: UALCAN: A portal for facilitating tumor subgroup gene expression and survival analyses. Neoplasia 19: 649-658, 2017.

23. Livak KJ and Schmittgen TD: Analysis of relative gene expression data using real-time quantitative PCR and the 2(-Delta Delta $\mathrm{C}(\mathrm{T}))$ method. Methods 25: 402-408, 2001.

24. Qian S, Han Y, Shi Y, Xu W, Zhu Y, Jiang S, Chen Y, Yu Z, Zhang S, Yang Y, et al: Benzene induces haematotoxicity by promoting deacetylation and autophagy. J Cell Mol Med 23: $1022-1033,2019$

25. Li XX, Guo H, Zhou JD, Wu DH, Ma JC, Wen XM, Zhang W, $\mathrm{Xu}$ ZJ, Lin J and Jun Q: Overexpression of CTNNB1: Clinical implication in Chinese de novo acute myeloid leukemia. Pathol Res Pract 214: 361-367, 2018

26. Petherick KJ, Williams AC, Lane JD, Ordóñez-Morán $P$, Huelsken J, Collard TJ, Smartt HJ, Batson J, Malik K, Paraskeva C and Greenhough A: Autolysosomal $\beta$-catenin degradation regulates Wnt-autophagy-p62 crosstalk. EMBO J 32: 1903-1916, 2013.

27. Hardie DG, Scott JW, Pan DA and Hudson ER: Management of cellular energy by the AMP-activated protein kinase system. FEBS Lett 546: 113-120, 2003.

28. Wang F, Song W, Zhao H, Ma Y, Li Y, Zhai D, Pi J, Si Y, Xu J, Dong L, et al: The RNA-binding protein QKI5 regulates primary miR-124-1 processing via a distal RNA motif during erythropoiesis. Cell Res 27: 416-439, 2017.

29. Huang X, Wu Z, Mei Y and Wu M: XIAP inhibits autophagy via XIAP-Mdm2-p53 signalling. EMBO J 32: 2204-2216, 2013.

30. Chae YB and Kim MM: Activation of $\mathrm{p} 53$ by spermine mediates induction of autophagy in HT1080 cells. Int J Biol Macromol 63 : 56-63, 2014.

31. Sui X, Jin L, Huang X, Geng S, He C and Hu X: p53 signaling and autophagy in cancer: A revolutionary strategy could be developed for cancer treatment. Autophagy 7: 565-571, 2011.
32. Järås M, Miller PG, Chu LP, Puram RV, Fink EC, Schneider RK, Al-Shahrour F, Peña P, Breyfogle LJ, Hartwell KA, et al: Csnk1a1 inhibition has p53-dependent therapeutic efficacy in acute myeloid leukemia. J Exp Med 211: 605-612, 2014.

33. Chen L, Li C, Pan Y and Chen J: Regulation of p53-MDMX interaction by casein kinase 1 alpha. Mol Cell Biol 25: 6509-6520, 2005.

34. Schittek B and Sinnberg T: Biological functions of casein kinase 1 isoforms and putative roles in tumorigenesis. Mol Cancer 13: 231, 2014

35. Richter J, Kretz AL, Lemke J, Fauler M, Werner JU, Paschke S, Leithäuser F, Henne-Bruns D, Hillenbrand A and Knippschild U: CK1 $\alpha$ overexpression correlates with poor survival in colorectal cancer. BMC Cancer 18: 140, 2018

36. Carrino M, Quotti Tubi L, Fregnani A, Canovas Nunes S, Barilà G, Trentin L, Zambello R, Semenzato G, Manni S and Piazza F: Prosurvival autophagy is regulated by protein kinase CK1 alpha in multiple myeloma. Cell Death Discov 5: 98, 2019.

37. Duran A, Amanchy R, Linares JF, Joshi J, Abu-Baker S, Porollo A, Hansen M, Moscat J and Diaz-Meco MT: p62 is a key regulator of nutrient sensing in the mTORC1 pathway. Mol Cell 44: 134-146, 2011.

38. Ma R, Zhang Y, Wang W, Wu J, Yang Q, Xu W, Jiang S, Han Y, Yu K and Zhang S: Inhibition of autophagy enhances the antitumour activity of tigecycline in multiple myeloma. J Cell Mol Med 22: 5955-5963, 2018.

39. Kojima K, Konopleva M, Samudio IJ, Schober WD, Bornmann WG and Andreeff M: Concomitant inhibition of MDM2 and $\mathrm{Bcl}-2$ protein function synergistically induce mitochondrial apoptosis in AML. Cell Cycle 5: 2778-2786, 2006.

40. Huart AS, MacLaine NJ, Meek DW and Hupp TR: CK1alpha plays a central role in mediating MDM2 control of p53 and E2F-1 protein stability. J Biol Chem 284: 32384-32394, 2009.

41. Perry ME, Piette J, Zawadzki JA, Harvey D and Levine AJ: The mdm-2 gene is induced in response to UV light in a p53-dependent manner. Proc Natl Acad Sci USA 90: 11623-11627, 1993.

(i) $\odot$ This work is licensed under a Creative Commons Attribution-NonCommercial-NoDerivatives 4.0 International (CC BY-NC-ND 4.0) License. 